\title{
Pelvic Tilt Cannot Be Accurately Predicted Using Anteroposterior Radiographs
}

\author{
Zlatan Cizmic MD ${ }^{1}$, Jonathan Gabor BS ${ }^{2}$, Nima Eftekhary $\mathrm{MD}^{2}$, Hayeem \\ Rudy BA ${ }^{2}$, Michael O'Sullivan $\mathrm{MD}^{3}$, Ran Schwarzkopf $\mathrm{MD}^{2}$, Aaron \\ Buckland $\mathrm{MD}^{2}$, Jonathan Vigdorchik $\mathrm{MD}^{4 *}$ \\ ${ }^{1}$ Ascension Providence Hospital \\ ${ }^{2}$ NYU Langone Health \\ ${ }^{3}$ Central DuPage Hospital \\ ${ }^{4}$ Hospital for Special Surgery \\ zlatancizmic91@gmail.com, Jonathan.gabor@nyulangone.org, \\ nima.eftekhary@nyulangone.org, daniel.waren@nyulangone.org, \\ mo'sullivan@nsosmc.com.au, ran.schwarzkopf@nyulangone.org, \\ aaron.buckland@nyulangone.org, and vigdorchikj@hss.edu
}

\begin{abstract}
INTRODUCTION: Pelvic positioning during total hip arthroplasty (THA) can affect the functional position of the acetabular component. A comprehensive understanding of pelvic orientation prior to THA is necessary to allow for proper cup positioning and mitigate the risks of complications associated with component malpositioning. Measurements using anteroposterior (AP) radiographs have been described as an effective means of accurately predicting pelvic functional orientation. The purpose of our study was to describe the accuracy of assessing pelvic tilt using AP radiographs alone.

METHODS: An online survey was created and sent to a cohort of fellowship-trained adult reconstruction surgeons. The survey consisted of 65 standing AP pelvis radiographs. Participants were asked to score each radiograph as 1) anterior pelvic tilt > 10 degrees, 2) posterior pelvic tilt $>10$ degrees, or 3) neutral. Responses were then compared to measurements of pelvic tilt made on lateral standing pelvic radiographs. Categorical and continuous variables were compared using chi-squared, unpaired, twotailed student's $\mathrm{T}$ tests, and
\end{abstract}

RESULTS: 135 surgeons completed the survey. The average correct predictive value of pelvic tilt between all surgeons was $53.2 \%$. 51.2\% of responding surgeons performed 
greater than 100 cases per year. $50 \%$ of surgeons reported that they were "not so familiar" or "somewhat familiar" with the principles of spinopelvic mobility. $43.5 \%$ of surgeons reported that they did not routinely use spinopelvic mobility principles in THA planning.

DISCUSSION: The standing AP pelvis radiograph is poorly predictive of pelvic tilt. Preoperative evaluation of spinopelvic parameters requires AP and lateral pelvic views for detailed assessment and accurate pre-operative planning.

\section{Introduction}

Spinopelvic mobility refers to the motion that occurs between the spine, pelvis, and hips between changes in body positioning to maintain sagittal alignment and upright balance [1]. The rotation that occurs at the pelvis in the sagittal plane between these positions as the pelvis rotates around the femoral heads is referred to as pelvic tilt [2]. The predictable effects of pelvic tilt on acetabular anteversion and inclination play an important role in total hip arthroplasty (THA). Lembeck et al. demonstrated that every degree of posterior pelvic tilt is associated with an approximate 0.7 degree increase of functional anteversion of the acetabulum [3].

The phenomenon of increased anteversion and inclination as the pelvis rotates posteriorly in the transition from standing to sitting is referred to as "the biological opening of the acetabulum." [4] This motion is necessary to avoid anterior impingement of the femoral neck in the flexed and internally rotated hip while in the seated position. It is often altered in patients with coexisting hip-spine pathology and corresponds with a seven-fold increase in the risk of dislocation within 12 months from an index THA compared to those with no spine pathology [5-9].

Two-dimensional, antero-posterior (AP) pelvic radiographs are considered by many hip surgeons to be the standard imaging modality for assessment of the hip in THA [1,10-12]. The use of lateral radiographs of the lumbar spine and pelvis to study postural change and spinopelvic mobility is a relatively new concept $[2,7,13]$. The standard use of AP pelvic radiographs has led to some attempts to correlate spinopelvic parameters such a pelvic tilt to measurable ratios visible on AP radiograph [1417]. Tannast et al. reported that a line drawn between the upper border of the pubic symphysis and the sacrococcygeal joint on AP radiographs has the strongest correlation with actual pelvic tilt measured on lateral radiographs [15]. Despite this and other attempts to correlate AP radiographic measurements with pelvic tilt, the degree to which spinopelvic mobility can be accurately assessed on AP pelvic radiographs in THA patients unknown.

The goals of our study were three-fold: 1.) determine the ability of surgeons, with varying levels of familiarity with spinopelvic concepts, to accurately measure Anterior Pelvic Plane tilt (APPt) based on AP pelvic radiographs alone; 2.) quantify the level of familiarity hip surgeons have with spinopelvic concepts; and 3.) quantify how frequently these concepts are applied in THA planning. We hypothesize

that APPt will be poorly predicted based on AP radiographs alone, and that, as a group, hip surgeons are only moderately familiar with spinopelvic mobility principles in THA.

\section{Methods}

An online survey was created and sent to an international cohort of fellowship-trained adult reconstructive surgeons in the United States (US) and Australia (AUS). The survey consisted of 65 consecutive standing AP pelvis radiographs. During radiographic imaging, patients were asked to retain a natural, relaxed posture. The mean age of the 65 patients was 62 years (range 55-72). Fifty-two percent were female. 
We defined an important predictive accuracy of pelvic tilt on AP radiographs to be approximately $76 \%$ [18]. The power was set to 0.85 and the overall type I error probability at 0.1 . Applying these assumptions yielded an approximate required sample of 38 subjects. To account for an approximate $20 \%$ lack of response, we queried 135 hip surgeons. Surveys were sent electronically to members of the Australian Arthroplasty Society in addition to a number of US surgeons. Participants were surveyed regarding the number of THAs performed annually, as well as familiarity with the subject of spinopelvic mobility as it relates to component placement in THA. Surgeons were also queried about whether they routinely used concepts related to spinopelvic mobility in planning for THA and their predominantly used surgical approach (Figure 1).

Participants were then asked to score each of the $65 \mathrm{AP}$ pelvis radiographs as 1) anterior pelvic tilt $>10$ degrees, 2) posterior pelvic tilt $>10$ degrees, or 3) neutral (Figure 1). Responses were then compared to control measurements performed in a previously described, standard fashion using lateral, standing pelvic radiographs for each patient (Figure 2) [19]. Radiographic control measurements were performed by a single hip surgeon highly experienced in spinopelvic mobility concepts using a digital radiographic measurement tool provided by the hospital picture archiving and communications system (PACS, New York, NY, USA). All lateral radiographs used as controls were measured prior to survey distribution. Results of APPt accuracy were available to the surgeon immediately after completion. 
Figure 1: Sample questionnaire answered by each surgeon and anteroposterior radiograph used to query surgeon ability to predict pelvic tilt

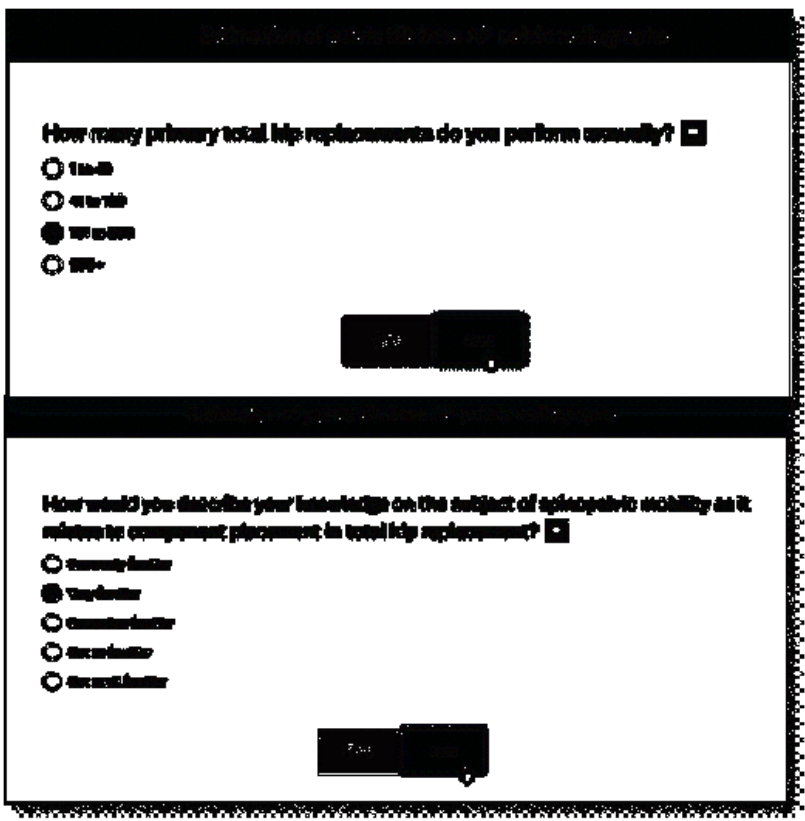

Putionte

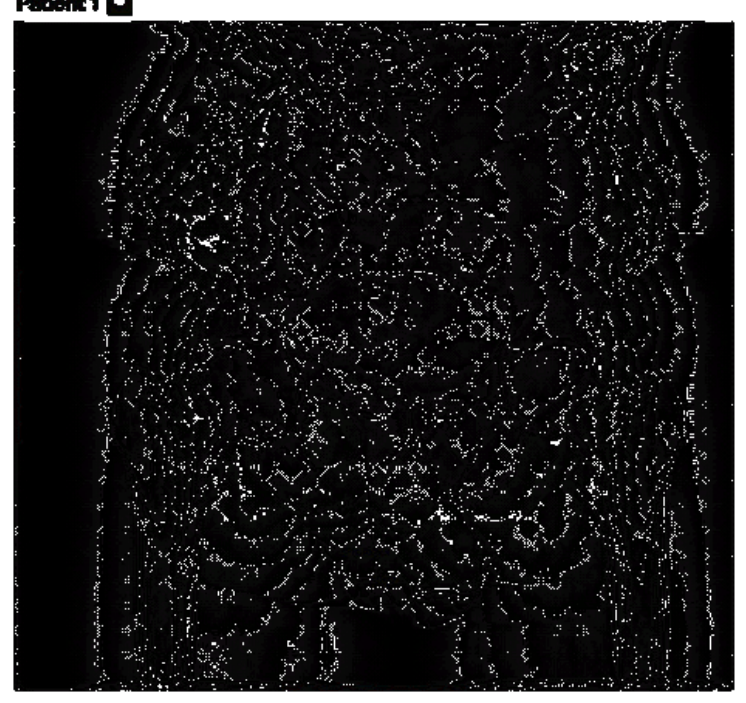

What is the patue tite of thit petionte

Onim

Onim

onere 
Figure 2: Lateral pelvic radiograph measurement used to obtain control values against which surgeons were graded

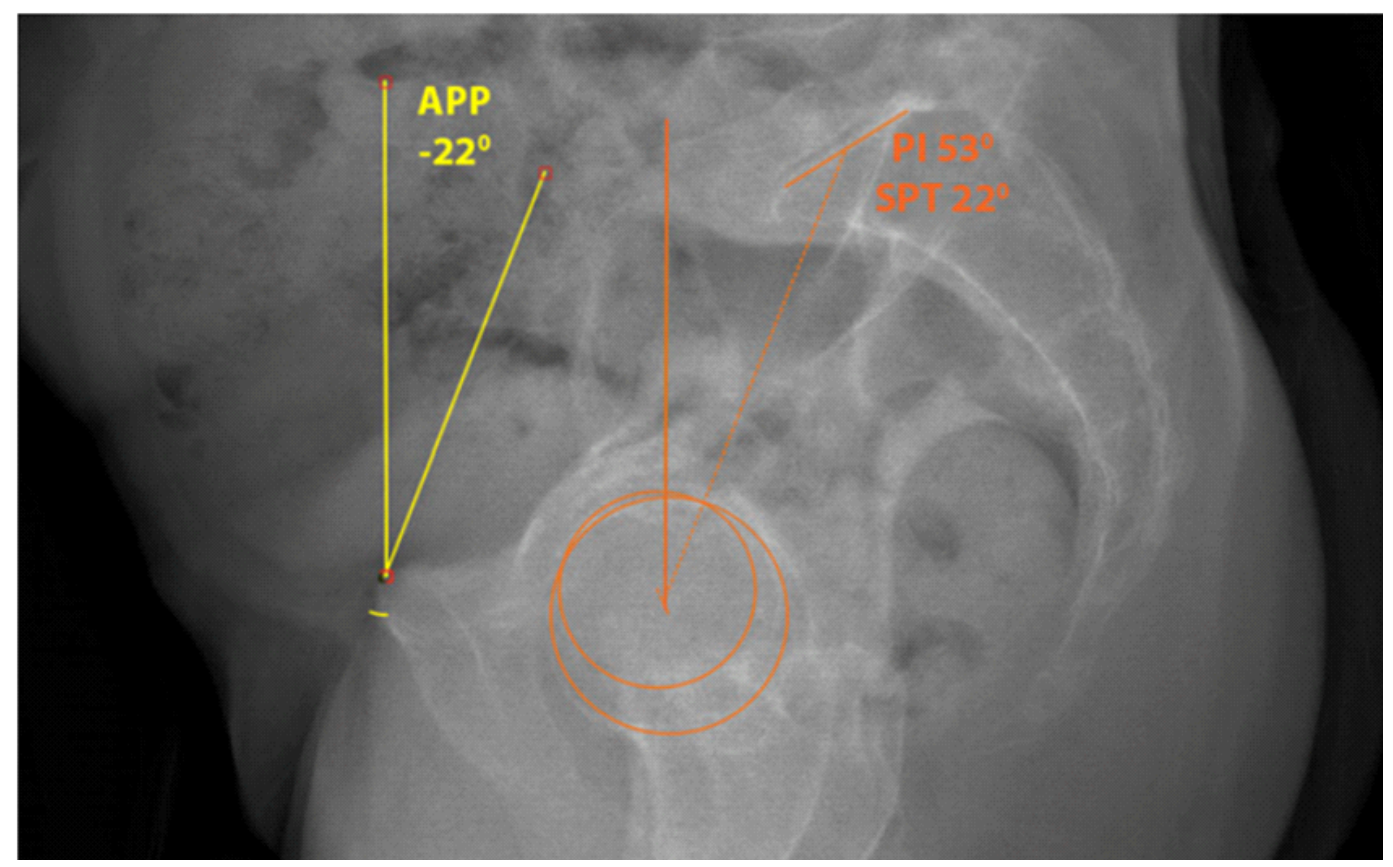

All descriptive and inferential statistics were conducted using SPSS v23 (International Business Machines, Armonk NY) statistics software. Two-tailed Student's t-tests were performed to compare means among continuous variables and ANOVA was used to compare scores amongst three or more groups. All tests performed were 2 -sided, with a p-value $<0.05$ deemed statistically significant. 


\section{Results}

There was a total of 135 respondents (101 respondents from the US and 34 from AUS). All respondents were fellowship-trained joint arthroplasty surgeons. The mean percentage correct of all respondents was $53.2 \%$ (range: $23 \%-68 \%$, SD $8.3 \%$ ) (Table 1 ).

\begin{tabular}{|c|c|c|c|}
\hline \multicolumn{4}{|c|}{ Table 1: Comparison of quiz score by surgeon practice, familiarity, and demographics } \\
\hline & $\mathrm{n}(\%) *$ & Score (SD) & $\mathrm{p}$-value** \\
\hline Country & & & 0.44 \\
\hline AUS & $34(25.2)$ & $52.7(9.1)$ & \\
\hline USA & $101(74.8)$ & $54.9(5.4)$ & \\
\hline Surgeon Volume & & & 0.72 \\
\hline $1-40$ & $8(5.9)$ & $54.6(4.7)$ & \\
\hline $41-100$ & $42(31.1)$ & $51.0(11.3)$ & \\
\hline $101-200$ & $48(35.6)$ & $54.1(6.0)$ & \\
\hline $201+$ & $37(27.4)$ & $53.4(10.2)$ & \\
\hline Predominant Surgical Approach & & & 0.10 \\
\hline Non-posterior & $22(15.9)$ & $58.2(3.8)$ & \\
\hline Posterior & $113(84.1)$ & $52.6(8.7)$ & \\
\hline Familiarity with spinopelvic mobility & & & 0.57 \\
\hline Not so familiar & $29(21)$ & $56.6(5.1)$ & \\
\hline Somewhat familiar & $42(31)$ & $52.3(6.4)$ & \\
\hline Very familiar & $39(29)$ & $52.3(10.4)$ & \\
\hline Extremely familiar & $25(19)$ & $52.3(10.1)$ & \\
\hline $\begin{array}{l}\text { Routine usage of spinopelvic mobility } \\
\text { in planning }\end{array}$ & & & 0.54 \\
\hline No & $66(49)$ & $52.4(10.7)$ & \\
\hline Yes & $69(51)$ & $54.9(6.0)$ & \\
\hline
\end{tabular}

The majority of surgeons performed between 41 and 200 hip replacement cases per year. Eight surgeons performed between 1 and 40 cases per year, and 37 surgeons performed greater than 200 cases per year. There was no significant association between surgeon volume and ability to predict APPt on AP radiograph $(\mathrm{p}=0.72)$. Eighty-four percent of surgeons $(\mathrm{n}=113)$ reported that they predominantly used a posterior surgical approach compared with a non-posterior approach. There was no significant association between predominant surgical approach and ability to predict $\mathrm{APPt}(\mathrm{p}=0.10)$.

Fifty-two percent of all surgeons reported being "not so familiar" or "somewhat familiar" with concepts relating to spinopelvic mobility. Twenty-nine percent reported being "very familiar" and 19\% reported being "extremely familiar" with these concepts. There was no significant association between familiarity with spinopelvic concepts and ability to predict APPt ( $\mathrm{p}=0.57)$.

Similarly, approximately half of all surgeon-respondents reported that they did not routinely use concepts of spinopelvic mobility in planning THA $(n=66,49 \%)$. There was no significant association however between routine usage of spinopelvic mobility planning in THA and the ability to accurately predict APPt on AP pelvic radiograph $(\mathrm{p}=0.54)$. 


\section{Discussion}

Fellowship-trained adult reconstructive surgeons can correctly predict APPt using an AP pelvic radiograph in only about $50 \%$ of cases. Higher case volumes, greater familiarity with spinopelvic mobility concepts, and more experience using principles of spinopelvic mobility in THA planning do not confer an advantage in assessing pelvic tilt from AP radiographs. Half of the responding surgeons describe themselves as "not so familiar" or only "somewhat familiar" with spinopelvic mobility principles, and roughly $40 \%$ of hip surgeons deny routine usage of spinopelvic mobility concepts in planning THA altogether. Our results suggest an underappreciation of these concepts and the need for further elucidation of the topic.

While lateral pelvic radiographs in the standing and seated positions should be used to calculate the change in pelvic tilt and assess for the presence of spinal stiffness or deformity that may predispose a patient to dislocation, they are not yet routinely used in clinical practice $[1,7,13,20-22]$. Rather, orthopaedic surgeons typically refer to AP radiographs for surgical planning; which alone are not routinely used for evaluating spinopelvic mobility [1,10-12]. Prior literature suggests that AP radiographs can be used to estimate spinopelvic parameters with some degree of reliability $[15,18,22]$. Tannast and colleagues sought to compare six previously described methods of estimating pelvic tilt on AP pelvic radiographs [15]. A moderately strong correlation was found between the vertical distance between the upper border of the pubic symphysis and the sacrococcygeal joint and pelvic tilt. The authors found that pelvic tilt could be estimated within 3.8 degrees for men and 4.2 degrees for women from this parameter. Blondel et al. investigated a different method for determining pelvic tilt in AP pelvic radiographs using the sacro-femoral-pubic angle (midpoint of S1 endplate to centroid of acetabuli to superior border of the pubic symphysis) [18]. A predictive ability of $76 \%$ accuracy was obtained $\left( \pm 7.5^{\circ}\right)$, demonstrating an improvement over the method reported by Tannast et al. More recently, Schwarz et al. explored the ratio between the height of the obturator foramen and the height of the lesser pelvis (defined as a line drawn from the lower sacroiliac joint to the upper border of the obturator foramen), as these distances change inversely with changes in pelvic tilt [22]. These ratios were estimated using an exponential formula calculated from simulated AP pelvic radiographs with pelvic tilt ranging from $+15^{\circ}$ to $-15^{\circ}$. This method yielded an accuracy within $3.9^{\circ}$, and the authors concluded that it was a suitable method for estimating pelvic tilt in daily clinical routine. However, the results of our study refute prior claims that pelvic tilt can be estimated on AP radiographs alone. In our study, the hip surgeons were able to predict APPt correctly in only $50 \%$ of cases, no more than a random flip of the coin. As such, the authors of the present study do not recommend that only a single AP radiograph be used in the preoperative workup of a patient undergoing THA, particularly in patients with possible spinal stiffness or deformity.

Although lateral radiographs are considered to be the gold standard modality for assessing APPt in patients undergoing THA, hip surgeons have been accustomed to assessing the acetabulum in a coronal plane using AP radiographs [1]. Traditional component positioning and postoperative assessment of THA has been based on coronal safe zones determined in the supine position [23]. However, the "Lewinnek safe zone", defined in the supine position, may not be applicable to patients in a functional, standing position due to the effects of pelvic tilt [1]. As a consequence, the concept of spinopelvic mobility and the use of lateral radiographs to assess sagittal motion of the hip have gained much attention since they were first described a decade ago $[7,13,24]$. Lazennec et al. performed pre- and post-operative standing EOS scans in 66 THA recipients and determined that, compared to the position of implants in the supine position, most acetabular and femoral components reside outside of the safe zone for patients in the standing position [25]. Recently, Pierrepont and colleagues characterized the variations in functional pelvic tilt between the supine, flexed-seated, and standing positions using lateral radiographs [26]. In a review of 4,042 consecutive patients undergoing THA, Langston et al. found that predictable spinopelvic mobility characteristics using lateral radiographs were correlated with increased risk of dislocation during certain motions postoperatively [27]. Their data underscores the importance 
of functional pelvic positioning and pelvic tilt during preoperative consultation for THA. Given this, the addition of lateral functional radiographs in the standing, sitting, and supine positions has been proposed as a critical tool for investigating spinopelvic mobility and functional pelvic positioning in THA candidates $[1,4,6,28]$. However, our study demonstrates that nearly $50 \%$ of hip surgeons are not familiar with spinopelvic mobility concepts and that roughly $40 \%$ of them do not include it in their preoperative workup. As the understanding of the hip-spine-pelvis relationship continues to grow, hip surgeons must continue to adapt, familiarize themselves with, and incorporate these concepts into their everyday practice.

Our study is not without limitations. First, this study was a subjective survey of hip surgeons with varying levels of experience with spinopelvic mobility. This may have led to some reporting bias and discrepancies in the accuracy of answers. However, the surveys were anonymous which may have led surgeons to answer more truthfully. Moreover, estimating APPt was limited to three answer choices that the surgeon could not inherently bias.

In our study, the use of AP radiographs alone for determining APPt was purposefully done to depict the lack of knowledge of spinopelvic interplay and was a key focus of the study. Furthermore, by limiting the use of lateral radiographs to serve only as controls against which the surgeon AP radiographic responses were compared further drives home the point that a single AP radiograph is an unacceptable method of determining APPt. The second limitation of our study includes a relatively small number of a participants and lower power which may underestimate the presence of true differences between surgeons and their ability to predict pelvic tilt with regards to differing annual case volumes, and ranges of familiarity and experience using spinopelvic mobility principles in THA. Despite these limitations, the low predictive accuracy, and poor levels of reported familiarity and utilization of spinopelvic principles in THA provide insight into the underappreciation of the topic. Furthermore, our power analysis yielded a sample size of 38 when using previously reported predictive accuracies of pelvic tilt using only AP radiographs. Future, large-scale, higher powered studies are needed to determine the ability of hip surgeons to accurately predict pelvic tilt in multiple radiographic planes, as well as any correlating factors which may influence this accuracy.

Our findings suggest that hip surgeons cannot accurately predict pelvic tilt based on AP radiographs alone. Given that many surgeons do not obtain lateral pelvic radiographs, the results of our study may describe a simple solution to decrease improper component positioning. Our findings also suggest that spinopelvic mobility and its role in THA may be underappreciated by many hip surgeons. Given the significant impact of coexisting hip-spine disease on THA outcomes, increased efforts are needed to promote the appreciation of this relevant topic in THA.

\section{References}

1. Ike H, Dorr LD, Trasolini N, Stefl M, McKnight B, Heckmann N. Spine-Pelvis-Hip Relationship in the Functioning of a Total Hip Replacement. J Bone Jt Surg 2018;100:1606-15. doi:10.2106/JBJS.17.00403.

2. Lazennec JY, Rousseau MA, Rangel A, Gorin M, Belicourt C, Brusson A, et al. Pelvis and total hip arthroplasty acetabular component orientations in sitting and standing positions: Measurements reproductibility with EOS imaging system versus conventional radiographies. Orthop Traumatol Surg Res 2011;97:373-80. doi:10.1016/J.OTSR.2011.02.006.

3. Lembeck B, Mueller O, Reize P, Wuelker N. Pelvic tilt makes acetabular cup navigation inaccurate. Acta Orthop 2005;76:517-23. doi:10.1080/17453670510041501.

4. Kanawade V, Dorr LD, Wan Z. Predictability of Acetabular Component Angular Change with Postural Shift from Standing to Sitting Position. J Bone Jt Surgery-American Vol 2014;96:978-86. doi:10.2106/JBJS.M.00765. 
5. DelSole EM, Vigdorchik JM, Schwarzkopf R, Errico TJ, Buckland AJ. Total Hip Arthroplasty in the Spinal Deformity Population: Does Degree of Sagittal Deformity Affect Rates of Safe Zone Placement, Instability, or Revision? J Arthroplasty 2017;32:1910-7. doi:10.1016/j.arth.2016.12.039.

6. Phan D, Bederman SS, Schwarzkopf R. The influence of sagittal spinal deformity on anteversion of the acetabular component in total hip arthroplasty. Bone Joint J 2015;97-B:1017-23. doi:10.1302/0301-620X.97B8.35700.

7. Lazennec J-Y, Brusson A, Rousseau M-A. Hip-spine relations and sagittal balance clinical consequences. Eur Spine J 2011;20:686. doi:10.1007/S00586-011-1937-9.

8. Buckland AJ, Puvanesarajah V, Vigdorchik J, Schwarzkopf R, Jain A, Klineberg EO, et al. Dislocation of a primary total hip arthroplasty is more common in patients with a lumbar spinal fusion. Bone Joint J 2017;99-B:585-91. doi:10.1302/0301-620X.99B5.BJJ-2016-0657.R1.

9. Perfetti DC, Schwarzkopf R, Buckland AJ, Paulino CB, Vigdorchik JM. Prosthetic Dislocation and Revision After Primary Total Hip Arthroplasty in Lumbar Fusion Patients: A Propensity Score Matched-Pair Analysis. J Arthroplasty 2017;32:1635-1640.e1. doi:10.1016/j.arth.2016.11.029.

10. Widmer K-H. A simplified method to determine acetabular cup anteversion from plain radiographs. J Arthroplasty 2004;19:387-90. doi:10.1016/J.ARTH.2003.10.016.

11. Pradhan R. Planar anteversion of the acetabular cup as determined from plain anteroposterior radiographs. J Bone Joint Surg Br 1999;81:431-5.

12. Fabeck L, Farrokh D, Tolley M, Descamps PY, Gebhart M, Delincé P. A method to measure acetabular cup anteversion after total hip replacement. Acta Orthop Belg 1999;65:485-91.

13. Lazennec JY, Riwan A, Gravez F, Rousseau MA, Mora N, Gorin M, et al. Hip spine relationships: Application to total hip arthroplasty. HIP Int., 2007.

14. Thorén B, Sahlstedt B. Influence of Pelvic Position on Radiographic Measurements of the Prosthetic Acetabular Component; An Experimental Study on a Pelvic Model. Acta Radiol 1990;31:133-6. doi:10.1177/028418519003100203.

15. Tannast M, Murphy SB, Langlotz F, Anderson SE, Siebenrock KA. Estimation of pelvic tilt on anteroposterior X-rays - a comparison of six parameters. Skeletal Radiol 2006;35:149-55. doi:10.1007/s00256-005-0050-8.

16. Kojima A, Nakagawa T, Tohkura A. Simulation of acetabular coverage of femoral head using anteroposterior pelvic radiographs. Arch Orthop Trauma Surg 1998;117:330-6.

17. Konishi N, Mieno T, D4 M, Japan A. Determination of Acetabular Coverage of the Femoral Head with Use of a Single Anteroposterior Radiograph A NEW COMPUTERIZED TECHNIQUE*. n.d.

18. Blondel B, Schwab F, Patel A, Demakakos J, Moal B, Farcy J-P, et al. Sacro-femoral-pubic angle: a coronal parameter to estimate pelvic tilt. Eur Spine J 2012;21:719-24. doi:10.1007/s00586-0112061-6.

19. Buckland A, DelSole E, George S, Vira S, Lafage V, Errico T, et al. Sagittal Pelvic Orientation A Comparison of Two Methods of Measurement. Bull Hosp Jt Dis 2017;75:234-40.

20. Gorin M, Roger B, Lazennec J-Y, Charlot N, Arafati N, Bissery A, et al. Hip-spine relationship: a radio-anatomical study for optimization in acetabular cup positioning. Surg Radiol Anat 2004;26:136-44. doi:10.1007/s00276-003-0195-x.

21. Luthringer TA, Vigdorchik JM. A Preoperative Workup of a "Hip-Spine" Total Hip Arthroplasty Patient: A Simplified Approach to a Complex Problem. J Arthroplasty 2019. doi:10.1016/J.ARTH.2019.01.012.

22. Schwarz T, Benditz A, Springorum H-R, Matussek J, Heers G, Weber M, et al. Assessment of pelvic tilt in anteroposterior radiographs by means of tilt ratios. Arch Orthop Trauma Surg 2018;138:1045-52. doi:10.1007/s00402-018-2931-z.

23. Lewinnek GE, Lewis JL, Tarr R, Compere CL, Zimmerman JR. Dislocations after total hipreplacement arthroplasties. J Bone Joint Surg Am 1978;60:217-20. 
24. Lazennec J-Y, Charlot N, Gorin M, Roger B, Arafati N, Bissery A, et al. Hip-spine relationship: a radio-anatomical study for optimization in acetabular cup positioning. Surg Radiol Anat 2004;26:136-44. doi:10.1007/s00276-003-0195-x.

25. Lazennec JY, Thauront F, Robbins CB, Pour AE. Acetabular and Femoral Anteversions in Standing Position are Outside the Proposed Safe Zone After Total Hip Arthroplasty. J Arthroplasty 2017;32:3550-6. doi:10.1016/j.arth.2017.06.023.

26. Pierrepont J, Hawdon G, Miles BP, Connor BO, Baré J, Walter LR, et al. Variation in functional pelvic tilt in patients undergoing total hip arthroplasty. Bone Joint J 2017;99-B:184-91. doi:10.1302/0301-620X.99B2.BJJ-2016-0098.R1.

27. Langston J, Pierrepont J, Gu Y, Shimmin A. Risk factors for increased sagittal pelvic motion causing unfavourable orientation of the acetabular component in patients undergoing total hip arthroplasty. Bone Joint J 2018;100-B:845-52. doi:10.1302/0301-620X.100B7.BJJ-20171599.R1.

28. Buckland AJ, Vigdorchik J, Schwab FJ, Errico TJ, Lafage R, Ames C, et al. Acetabular Anteversion Changes Due to Spinal Deformity Correction: Bridging the Gap Between Hip and Spine Surgeons. J Bone Joint Surg Am 2015;97:1913-20. doi:10.2106/JBJS.O.00276. 